Quantum Information and Computation, Vol. 16, No. 5\&6 (2016) 0423-0434

(C) Rinton Press

\title{
CONSTRUCTIONS OF $q$-ARY ENTANGLEMENT-ASSISTED QUANTUM MDS CODES WITH MINIMUM DISTANCE GREATER THAN $q+1$
}

\author{
JIHAO FAN \\ Department of Computer Science and Engineering, Southeast University \\ Nanjing, Jiangsu 211189, China \\ fanjh12@seu. edu. cn \\ HANWU CHEN \\ Department of Computer Science and Engineering, Southeast University \\ Nanjing, Jiangsu 211189, China \\ $h w_{-}$chen@seu. edu. cn \\ JUAN XU \\ College of Computer Science and Technology, Nanjing University of Aeronautics and Astronautics \\ Nanjing, Jiangsu 210016, China \\ juanxu@nuaa. edu.cn \\ Received (received date) \\ Revised (revised date)
}

\begin{abstract}
The entanglement-assisted stabilizer formalism provides a useful framework for constructing quantum error-correcting codes (QECC), which can transform arbitrary classical linear codes into entanglement-assisted quantum error correcting codes (EAQECCs) by using pre-shared entanglement between the sender and the receiver. In this paper, we construct five classes of entanglement-assisted quantum MDS (EAQMDS) codes based on classical MDS codes by exploiting one or more pre-shared maximally entangled states. We show that these EAQMDS codes have much larger minimum distance than the standard quantum MDS (QMDS) codes of the same length, and three classes of these EAQMDS codes consume only one pair of maximally entangled states.
\end{abstract}

Keywords: Entanglement-assisted quantum error-correcting codes, Quantum errorcorrecting codes, Maximal-distance-separable (MDS) codes, Maximally entangled state Communicated by: to be filled by the Editorial

\section{Introduction}

Quantum error-correcting codes (QECC) play a key role in protecting quantum information from decoherence and quantum noise. The theory of quantum stabilizer codes allows one to import classical additive codes that satisfy certain dual-containing relationship for use as a QECC 1, 2, 3. Recently, a more general framework called entanglement-assisted stabilizer formalism was developed to construct QECCs with the help of pre-shared entanglement between the sender and the receiver [4. This framework has the advantage that it allows to construct QECCs from arbitrary classical linear codes, without the dual-containing constraint. Currently, many works have focused on the construction of binary EAQECCs based on classical binary or quaternary linear codes, see [5, 6, 7, 8, 9, 10, since binary QECCs might be the 
most useful ones in the future quantum computers and quantum communications. However, nonbinary cases have received less attention. Nonbinary EAQECCs would be useful in some quantum communication protocols [11, 12. Just as in the classical error-correcting codes (ECC) and standard QECCs, EAQECCs over higher alphabets can be used for constructing easily decodable binary EAQECCs by using concatenation technology [13, 14. Furthermore, nonbinary QECCs and EAQECCs, especially nonbinary quantum MDS (QMDS) codes and entanglement-assisted quantum MDS (EAQMDS) codes, are of significantly theoretical interest, since QMDS codes and EAQMDS codes can achieve the quantum Singleton bound [3] and the entanglement-assisted quantum Singleton bound [4, respectively.

Let $q$ be a prime power. We use $\mathcal{Q}=[[n, k, d]]_{q}$ to denote a standard $q$-ary QECC of length $n$ with size $q^{k}$ and minimum distance $d$. Then $\mathcal{Q}$ is a $q^{k}$-dimensional subspace of the $q^{n}$-dimensional Hilbert space $\left(\mathbb{C}^{q}\right)^{\otimes n}$, which can detect up to $d-1$ and correct up to $\lfloor(d-1) / 2\rfloor$ quantum errors. The parameters of $\mathcal{Q}$ have to satisfy the quantum Singleton bound: $k \leq n-2 d+2$ in [3]. If $\mathcal{Q}$ attains the quantum Singleton bound, then it is called a quantum maximum-distance-separable (MDS) code. According to the MDS conjecture in [3], the maximal length of a QMDS code cannot exceed $q^{2}+1$, i.e., $n \leq q^{2}+1$, except for the trivial and some special cases in [15, and except for the existence of QMDS codes with parameters $\left[\left[q^{2}+2, q^{2}-4,4\right]\right]_{q}$ for $q=2^{m}$ shown in [16]. As mentioned in [17, QMDS codes of length up to $q+1$ have been constructed for all possible dimensions, see [18, 19]. However, the problem of constructing QMDS codes with length $n$ greater than $q+1$ is much more difficult. Many QMDS codes with certain lengths between $q+1$ and $q^{2}+1$ have been obtained, see 20, 21, 22, 23, 24, 25, 26, 27. Up to now, the minimum distance of all known nontrivial $q$-ary QMDS codes is less than or equal to $q+1$, except for a few sporadic QMDS codes with large minimum distance in [16. It seems very difficult to improve this limit by using the standard Euclidean or Hermitian construction.

Inspired by these works, in this paper, we propose several constructions of EAQMDS codes based on classical MDS codes, and we get new $q$-ary EAQMDS codes with minimum distance greater than $q+1$ for some certain code lengths, while consuming a few pre-shared maximally entangled states. If we denote a $q$-ary EAQECC by $[[n, k, d ; c]]_{q}$, where $c$ is the number of maximally entangled states required, we get five classes of EAQMDS codes with parameters:

(i) $\left[\left[q^{2}+1, q^{2}-2 d+4, d ; 1\right]\right]_{q}$, where $q$ is a prime power, $2 \leq d \leq 2 q$ is an even integer.

(ii) $\left[\left[q^{2}, q^{2}-2 d+3, d ; 1\right]\right]_{q}$, where $q$ is a prime power, $q+1 \leq d \leq 2 q-1$.

(iii) $\left[\left[q^{2}-1, q^{2}-2 d+2, d ; 1\right]\right]_{q}$, where $q$ is a prime power, $2 \leq d \leq 2 q-2$.

(iv) $\left[\left[\frac{q^{2}-1}{2}, \frac{q^{2}-1}{2}-2 d+4, d ; 2\right]\right]_{q}$, where $q$ is an odd prime power, $\frac{q+1}{2}+2 \leq d \leq \frac{3}{2} q-\frac{1}{2}$.

(v) $\left.\left[\frac{q^{2}-1}{t}, \frac{q^{2}-1}{t}-2 d+t+2, d ; t\right]\right]_{q}$, where $q$ is an odd prime power with $t \mid(q+1), t \geq 3$ is an odd integer, and $\frac{(t-1)(q+1)}{t}+2 \leq d \leq \frac{(t+1)(q+1)}{t}-2$.

EAQMDS codes in (i)-(v) have minimum distance upper limit greater than $q+1$ by consuming a few pre-shared maximally entangled states. In particular, each code in (i)-(iii) has nearly double minimum distance upper limit of the standard QMDS code of the same length constructed so far, and consumes only one pair of maximally entangled states. This means that these codes have much better error-correction abilities than the standard QMDS codes of the same length and consume little entanglement. 
This paper is organized as follows. In Section 2, we introduce some basic notations and definitions of classical ECCs and EAQECCs. We propose several constructions of EAQMDS codes in Section 3. The conclusion is given in Section 4.

\section{Preliminaries}

Firstly, we review some basic results of classical RS codes, constacyclic codes and several formulas for EAQECCs. For details on classical ECCs and EAQECCs, see the literature 13, 28, and 4, 11, 29, respectively.

Let $p$ be a prime number and $q$ a power of $p$, i.e., $q=p^{r}$ for some $r>0 . \mathbb{F}_{q^{2}}$ denotes the finite field with $q^{2}$ elements. For any $a \in \mathbb{F}_{q^{2}}$, we denote by $\bar{a}=a^{q}$ the conjugation of $a$. For two vectors $\mathbf{x}=\left(x_{1}, x_{2}, \ldots, x_{n}\right)$ and $\mathbf{y}=\left(y_{1}, y_{2}, \ldots, y_{n}\right) \in \mathbb{F}_{q^{2}}^{n}$, their Hermitian inner product is defined as

$$
\langle\mathbf{x}, \mathbf{y}\rangle_{h}=\sum_{i=1}^{n} \overline{x_{i}} y_{i}=\overline{x_{1}} y_{1}+\overline{x_{2}} y_{2}+\cdots+\overline{x_{n}} y_{n} .
$$

Let $\mathcal{C}=[n, k]$ be a $q^{2}$-ary linear code of length $n$ and dimension $k$. The Hermitian dual code of $\mathcal{C}$ is defined as

$$
\mathcal{C}^{\perp_{h}}=\left\{\mathbf{x} \in \mathbb{F}_{q^{2}}^{n} \mid\langle\mathbf{x}, \mathbf{y}\rangle_{h}=0, \forall \mathbf{y} \in \mathcal{C}\right\} .
$$

If $\mathcal{C} \subseteq \mathcal{C}^{\perp_{h}}$, then $\mathcal{C}$ is called a Hermitian self-orthogonal code. On the contrary, if $\mathcal{C}^{\perp_{h}} \subseteq \mathcal{C}$, then $\mathcal{C}$ is called a Hermitian dual-containing code. Let $H=\left(a_{i j}\right)_{(n-k) \times n}$ be the parity check matrix of $\mathcal{C}$ over $\mathbb{F}_{q^{2}}$ with indices $1 \leq i \leq n-k$ and $1 \leq j \leq n$, then the Hermitian conjugate of $H$ is defined as

$$
H^{\dagger}=\left(\overline{a_{j i}}\right)_{n \times(n-k)},
$$

where the dagger $(\dagger)$ denotes the conjugate transpose operation over matrices in $\mathbb{F}_{q^{2}}$.

A Reed-Solomon code (denoted by $\mathcal{R S}(n, r)$ ) over $\mathbb{F}_{q^{m}}$ is a cyclic code of length $n=q^{m}-1$ with roots $\alpha, \alpha^{2}, \ldots, \alpha^{r-1}$, where $r$ is an integer with $1 \leq r \leq n-2, \alpha$ is a primitive element of $\mathbb{F}_{q^{m}}$. Its generator polynomial is $g(x)=(x-\alpha)\left(x-\alpha^{2}\right) \cdots\left(x-\alpha^{r-1}\right)$. The parameters of $\mathcal{R S}(n, r)$ are $[n, k, d]_{q^{m}}$, where $k=n-r+1, d=r$. The parity check matrix of $\mathcal{R S}(n, r)$ is given by

$$
H_{\mathcal{R S}(n, r)}=\left(\begin{array}{cccc}
1 & \alpha & \cdots & \alpha^{n-1} \\
1 & \alpha^{2} & \cdots & \alpha^{2(n-1)} \\
\vdots & \vdots & \vdots & \vdots \\
1 & \alpha^{r-1} & \cdots & \alpha^{(r-1)(n-1)}
\end{array}\right) .
$$

Let $\lambda$ be a nonzero element of $\mathbb{F}_{q^{2}}$, then a linear code $\mathcal{C}$ of length $n$ over $\mathbb{F}_{q^{2}}$ is said to be $\lambda$-constacyclic if $\left(\lambda c_{n}, c_{1}, \ldots, c_{n-1}\right) \in \mathcal{C}$ for every $\left(c_{1}, c_{2}, \ldots, c_{n}\right) \in \mathcal{C}$. If $\lambda=1, \mathcal{C}$ is a cyclic code. If $\lambda=-1, \mathcal{C}$ is called a negacyclic code. We assume that $\operatorname{gcd}\left(n, q^{2}\right)=1$. A codeword $\left(c_{1}, c_{2}, \ldots, c_{n}\right) \in \mathcal{C}$ is identified with its polynomial representation $c(x)=c_{0}+$ $c_{1} x+\cdots+c_{n-1} x^{n-1}$. It is easy to find that a $\lambda$-constacyclic code $\mathcal{C}$ of length $n$ over $\mathbb{F}_{q^{2}}$ is an ideal of the quotient ring $\mathbb{F}_{q^{2}}[x] /\left\langle x^{n}-\lambda\right\rangle$. It is known that $\mathcal{C}$ is generated by a monic divisor $g(x)$ of $x^{n}-\lambda$. The polynomial $g(x)$ is called the generator polynomial of the code $\mathcal{C}$. Let $\lambda \in \mathbb{F}_{q^{2}}$ be a primitive $r$ th root of unity. Let $\eta$ denote a primitive $r n$th root of unity (exists in some extension field) such that $\eta^{n}=\lambda$. Let $\zeta=\eta^{r}$ be a primitive $n$th root of 
unity. It follows from [30] that the roots of $x^{n}-\lambda$ are $\left\{\eta \zeta^{i}=\eta^{1+r i} \mid 0 \leq i \leq n-1\right\}$. Denote $\Omega=\{1+r i \mid 0 \leq i \leq n-1\}$. Then the defining set of a $\lambda$-constacyclic code $\mathcal{C}$ with generator polynomial $g(x)$ is $Z=\left\{i \in \Omega \mid g\left(\eta^{i}\right)=0\right\}$. It is easy to see that the defining set $Z$ is a union of some $q^{2}$-cyclotomic cosets modulo $r n$. There exist the following BCH bound for cyclic codes and the generalized $\mathrm{BCH}$ bound for $\lambda$-constacyclic codes.

Lemma 1 ([13], Ch.7) Let $\mathcal{C}$ be a cyclic code of length $n$ over $\mathbb{F}_{q^{2}}$. Let $\alpha \in \mathbb{F}_{q^{2}}$ be a primitive $n$-th root of unity. Suppose that $\mathcal{C}$ has generator polynomial $g(x)$ such that for some integers $b \geq 0$ and $\delta \geq 1, g\left(\alpha^{b}\right)=g\left(\alpha^{b+1}\right)=\cdots=g\left(\alpha^{b+\delta-2}\right)=0$, that is, the code has a string of $\delta-1$ consecutive powers of $\alpha$ as zeros. Then the minimum distance of $\mathcal{C}$ is at least $\delta$.

Lemma 2 (30], Lemma 4) Let $\mathcal{C}$ be a $\lambda$-constacyclic code of length $n$ over $\mathbb{F}_{q^{2}}$, where $\lambda \in \mathbb{F}_{q^{2}}$ is a primitive rth root of unity. Suppose that the generator polynomial $g(x)$ of $\mathcal{C}$ has the elements $\left\{\eta^{1+r i} \mid i_{0} \leq i \leq i_{0}+d-2\right\}$ as roots, where $\eta$ is a primitive rnth root of unity, $i_{0}$ is an integer. Then the minimum distance of $\mathcal{C}$ is at least $d$.

The following lemma gives a sufficient and necessary condition for a $q^{2}$-ary $\lambda$-constacyclic code to be Hermitian dual-containing.

Lemma 3 (24], Lemma 2.2) Let $\mathcal{C}$ be a $\lambda$-constacyclic code of length $n$ over $\mathbb{F}_{q^{2}}$ with defining set $Z$ and let $\lambda \in \mathbb{F}_{q^{2}}$ be a primitive rth root of unity. Then $\mathcal{C}$ is a Hermitian dual-containing code if and only if $Z \cap Z^{-q}=\emptyset$ where $Z^{-q}=\{-q z(\bmod r n) \mid z \in Z\}$.

An $[[n, k, d ; c]]_{q}$ EAQECC encodes $k$ information qudits into $n$ channel qudits with the help of $c$ pairs of maximally entangled states. The minimum distance is $d$. One of the focuses of the construction of EAQECCs is to determine the number of maximally entangled pairs required for the encoding. For example, the optimal number of entangled pairs required by an arbitrary binary EAQECC is given in 29 .

Theorem 1 (29], Theorem 1) Suppose that an EAQECC is constructed from generators corresponding to the rows in a quantum check matrix

$$
H=\left[H_{Z} \mid H_{X}\right],
$$

where $H$ is an $[(n-k) \times 2 n]$-dimensional binary matrix representing the quantum code (see [2, 31] ), and both $H_{Z}$ and $H_{X}$ are $[(n-k) \times n]$-dimensional binary matrices. Then the resulting code is an $[n, k+c ; c]$ entanglement-assisted code and requires $c$ ebits, where

$$
c=\operatorname{rank}\left(H_{X} H_{Z}^{T}+H_{Z} H_{X}^{T}\right) / 2
$$

and addition is binary.

Several formulas for different EAQECCs are given as corollaries in 29. Similar results are also available for nonbinary EAQECCs. According to [29, a formula similar to (2) holds for $q$-ary EAQECCs by using $q$-dimensional entangled pairs. The number of the corresponding entangled pairs is given by

$$
c=\operatorname{rank}\left(H_{X} H_{Z}^{T}-H_{Z} H_{X}^{T}\right) / 2
$$

and subtraction is in the finite field $\mathbb{F}_{q}$. There are the following corollaries for general EAQECCs.

Corollary 1 ([29]) Let $H$ be the parity check matrix of an $[n, k, d]_{q^{2}}$ classical linear code over $\mathbb{F}_{q^{2}}$. Then an $[[n, 2 k-n+c, d ; c]]_{q} E A Q E C C$ can be obtained, where $c=\operatorname{rank}\left(H H^{\dagger}\right)$ is the number of maximally entangled states required. 
Corollary 2 (EA-Singleton Bound, [4]) An $[[n, k, d ; c]]_{q}$ EAQECC satisfies

$$
n+c-k \geq 2(d-1)
$$

where $0 \leq c \leq n-1$.

\section{Constructions of $q$-ary EAQMDS codes}

A classical linear MDS code can lead to an EAQECC that meets the corresponding EASingleton bound [4. The main task is to determine the number of maximally entangled pairs that required. For the $q$-ary QMDS code of length $n$, the construction problem has been completely solved when length $n \leq q+1$, see [18, 19. Therefore, we do not need to consume extra entanglement resources for the construction when length $n \leq q+1$. However, the introduction of a certain amount of pre-shared entanglement is useful for the case when length $n>q+1$, since we may have more variety for the parameters of EAQMDS codes than those of standard QMDS codes.

\subsection{EAQMDS codes based on cyclic MDS codes}

We take $\mathcal{C}$ as a $q^{2}$-ary cyclic code over $\mathbb{F}_{q^{2}}$ of length $n$, where $n \mid q^{2}+1$. Then the $q^{2}$ cyclotomic coset modulo $n$ containing $i$ is denoted by $C_{i}=\left\{i, i q^{2}, i q^{4}, \ldots, i q^{2\left(m_{i}-1\right)}\right\}$, where $m_{i}$ is the smallest positive integer such that $q^{m_{i}} i=i(\bmod n)$. The following result gives the $q^{2}$-cyclotomic cosets modulo $n$.

Lemma 4 ([22], Lemma 4.1) Let $n \mid q^{2}+1$ and let $s=\left\lfloor\frac{n}{2}\right\rfloor$. If $n$ is odd, then the $q^{2}$-cyclotomic cosets modulo $n$ containing integers from 0 to $n$ are: $C_{0}=\{0\}, C_{i}=\{i,-i\}=\{i, n-i\}$, where $1 \leq i \leq s$. If $n$ is even, then the $q^{2}$-cyclotomic cosets modulo $n$ containing integers from 0 to $n$ are: $C_{0}=\{0\}, C_{s}=\{s\}$ and $C_{i}=\{i,-i\}=\{i, n-i\}$, where $1 \leq i \leq s-1$.

Lemma 5 Let $n \mid q^{2}+1$ and $s=\left\lfloor\frac{n}{2}\right\rfloor$. Let $\mathcal{C}$ be a $q^{2}$-ary cyclic code of length $n$ with defining set $Z=\cup_{i=0}^{\delta} C_{i}$, where $1 \leq \delta \leq \delta_{\max }=\left\lfloor\frac{n}{q+1}\right\rfloor$, and let $H$ be the parity check matrix of $\mathcal{C}$ over $\mathbb{F}_{q^{2}}$, then $\operatorname{rank}\left(H H^{\dagger}\right)=1$.

Proof. We divide the defining set $Z$ of $\mathcal{C}$ into two mutually disjoint subsets, i.e., $Z=C_{0} \cup Z_{1}$, where $Z_{1}=\cup_{i=1}^{\delta} C_{i}$. Let $\mathcal{C}_{1}$ be a $q^{2}$-ary cyclic code of length $n$ with $\operatorname{defining}$ set $Z_{1}$. We show $\mathcal{C}_{1}^{\perp_{h}} \subseteq \mathcal{C}_{1}$. Suppose that $\mathcal{C}_{1}$ is not a Hermitian dual-containing code, then $Z_{1} \cap Z_{1}^{-q} \neq \emptyset$ by Lemma 3. There exist $i$ and $j$, where $1 \leq i, j \leq \delta_{\max }$, such that $i=-q j(\bmod n)$ or $i=q j$ $(\bmod n)$. If the first case holds, it follows that $q+1 \leq i+q j<n$, which is a contradiction. If the second case holds, it follows that $1 \leq i \leq \delta_{\max }<q \leq q j \leq q \delta_{\max }<n$, which is also a contradiction. Therefore, we have $\mathcal{C}_{1}^{\perp_{h}} \subseteq \mathcal{C}_{1}$. Let the parity check matrix of $\mathcal{C}_{1}$ over $\mathbb{F}_{q^{2}}$ be $H_{1}$, then $H_{1} H_{1}^{\dagger}=0$. It is easy to see that the parity check matrix of $\mathcal{C}$ over $\mathbb{F}_{q^{2}}$ is given by $H=\left(\begin{array}{c}h_{0} \\ H_{1}\end{array}\right)$, where $h_{0}=(1,1, \ldots, 1)$. Since $n \mid q^{2}+1$, then we have $h_{0} h_{0}^{\dagger} \neq 0$. It is obvious that $C_{0} \cap Z_{1}^{-q}=\emptyset$, and it follows that $h_{0} H_{1}^{\dagger}=0$. Therefore, the rank of $H H^{\dagger}$ is equal to 1 .

Theorem 2 Let $n \mid q^{2}+1$. There exists an EAQMDS code with parameters

$$
[[n, n-2 d+3, d ; 1]]_{q}
$$

where $2 \leq d \leq 2\left\lfloor\frac{n}{q+1}\right\rfloor+2$ is an even integer. 
Proof. Let $\mathcal{C}$ be a cyclic code of length $n$ with defining set $Z=\cup_{i=0}^{\delta} C_{i}$, where $0 \leq \delta \leq$ $\delta_{\max }=\left\lfloor\frac{n}{q+1}\right\rfloor$. From Lemma 4, we know that the defining set $Z$ consists of $2 \delta+1$ consecutive integers $\{-\delta,-\delta+1, \ldots,-1,0,1, \ldots, \delta-1, \delta\}$. Then the dimension of $\mathcal{C}$ is $\operatorname{dim} \mathcal{C}=n-2 \delta-1$. From the $\mathrm{BCH}$ bound for cyclic codes in Lemma 1, we know that the minimum distance of $\mathcal{C}$ is at least $2 \delta+2$. Then $\mathcal{C}$ has parameters $[n, n-2 \delta-1, \geq 2 \delta+2]_{q^{2}}$. Combining Corollary 1. Lemma 5 and the EA-Singleton bound, we can obtain an EAQMDS code with parameters $[[n, n-4 \delta-1,2 \delta+2 ; 1]]_{q}$. Let $d=2 \delta+2$, then we have $2 \leq d \leq 2 \delta_{\max }+2=2\left\lfloor\frac{n}{q+1}\right\rfloor+2$.

Let $n=q^{2}+1$, then we can get the following EAQMDS code with minimum distance greater than $q+1$.

Corollary 3 There exists an EAQMDS code with parameters

$$
\left[\left[q^{2}+1, q^{2}-2 d+4, d ; 1\right]\right]_{q},
$$

where $q$ is a prime power, $2 \leq d \leq 2 q$ is an even integer.

Example 1 Let $q=4$, then $n=q^{2}+1=17$. Applying Corollary 3, we get two EAQMDS codes with minimum distance greater than $q+1=5$ whose parameters are $[[17,8,6 ; 1]]_{4}$, $[[17,4,8 ; 1]]_{4}$.

If we consider cyclic codes whose lengths satisfy $n \mid q^{2}-1$, then the corresponding $q^{2}$ cyclotomic coset modulo $n$ containing $i$ is $C_{i}=\{i\}, 0 \leq i \leq n-1$.

Lemma 6 Let $n \mid q^{2}-1$. Let $\mathcal{C}$ be a $q^{2}$-ary cyclic code of length $n$ with defining set $Z=$ $\cup_{i=-\delta}^{\delta} C_{i}$, where $1 \leq \delta \leq \delta_{\max }=\left\lfloor\frac{n}{q+1}\right\rfloor-1$, and let $H$ be the parity check matrix of $\mathcal{C}$ over $\mathbb{F}_{q^{2}}$, then $\operatorname{rank}\left(H H^{\dagger}\right)=1$.

Proof. We divide the defining set $Z$ of $\mathcal{C}$ into three mutually disjoint subsets, i.e., $Z=$ $Z_{1} \cup C_{0} \cup Z_{2}$, where $Z_{1}=\cup_{i=-\delta}^{-1} C_{i}$ and $Z_{2}=\cup_{i=1}^{\delta} C_{i}$. Let $\mathcal{C}_{1}$ and $\mathcal{C}_{2}$ be two $q^{2}$-ary cyclic codes of length $n$ with defining sets $Z_{1}$ and $Z_{2}$, respectively. It is easy to verify that there are $\mathcal{C}_{1}^{\perp_{h}} \subseteq \mathcal{C}_{1}, \mathcal{C}_{2}^{\perp_{h}} \subseteq \mathcal{C}_{2}$ and $\mathcal{C}_{1}^{\perp_{h}} \subseteq \mathcal{C}_{2}$. Let the parity check matrices of $\mathcal{C}_{1}$ and $\mathcal{C}_{2}$ over $\mathbb{F}_{q^{2}}$ be $H_{1}$ and $H_{2}$, respectively, then we have $H_{1} H_{1}^{\dagger}=0, H_{2} H_{2}^{\dagger}=0$ and $H_{1} H_{2}^{\dagger}=0$. Then the parity check matrix of $\mathcal{C}$ over $\mathbb{F}_{q^{2}}$ is given by $H=\left(\begin{array}{c}H_{1} \\ h_{0} \\ H_{2}\end{array}\right)$, where $h_{0}=(1,1, \ldots, 1)$. Since $n \mid q^{2}-1$, then we have $h_{0} h_{0}^{\dagger} \neq 0$. It is obvious that $C_{0} \cap Z_{1}^{-q}=\emptyset$ and $C_{0} \cap Z_{2}^{-q}=\emptyset$, and it follows that $h_{0} H_{1}^{\dagger}=0$ and $h_{0} H_{2}^{\dagger}=0$. Therefore, the rank of $H H^{\dagger}$ is equal to 1 .

Theorem 3 Let $n \mid q^{2}-1$. There exists an EAQMDS code with parameters

$$
[[n, n-2 d+3, d ; 1]]_{q},
$$

where $2 \leq d \leq 2\left\lfloor\frac{n}{q+1}\right\rfloor$

Proof. Let $\mathcal{C}$ be a cyclic code of length $n$ with defining set $Z=\cup_{i=-\delta}^{\delta} C_{i}$, where $0 \leq \delta \leq$ $\delta_{\max }=\left\lfloor\frac{n}{q+1}\right\rfloor-1$. Then the defining set $Z$ which consists of $2 \delta+1$ consecutive integers is given by $\{-\delta,-\delta+1, \ldots,-1,0,1, \ldots, \delta-1, \delta\}$. Therefore, $\operatorname{dim} \mathcal{C}=n-2 \delta-1$, and the minimum distance of $\mathcal{C}$ is at least $2 \delta+2$ by Lemma 1. Then $\mathcal{C}$ has parameters $[n, n-2 \delta-1, \geq 2 \delta+2]_{q^{2}}$. Combining Corollary 1, Lemma 6 and the EA-Singleton bound, we can obtain an EAQMDS code with parameters $[[n, n-4 \delta-1,2 \delta+2 ; 1]]_{q}$, where $2 \leq 2 \delta+2 \leq 2 \delta_{\max }+2=2\left\lfloor\frac{n}{q+1}\right\rfloor$. In order to get EAQMDS codes with odd minimum distance, we take the defining set of $\mathcal{C}$ as $Z=\cup_{i=-\delta^{\prime}}^{\delta^{\prime}-1} C_{i}$, where $1 \leq \delta^{\prime} \leq \delta_{\max }=\left\lfloor\frac{n}{q+1}\right\rfloor-1$. Then we can obtain an EAQMDS code with parameters $\left[\left[n, n-4 \delta^{\prime}+1,2 \delta^{\prime}+1 ; 1\right]\right]_{q}$, where $3 \leq 2 \delta^{\prime}+1 \leq 2 \delta_{\max }+1=2\left\lfloor\frac{n}{q+1}\right\rfloor-1$. 
Corollary 4 There exists an EAQMDS code with parameters

$$
\left[\left[q^{2}-1, q^{2}-2 d+2, d ; 1\right]\right]_{q},
$$

where $q$ is a prime power, $2 \leq d \leq 2 q-2$ is an integer.

Example 2 Let $q=5$, then $n=q^{2}-1=24$. Applying Corollary 4 , we get four EAQMDS codes with minimum distance greater than $q-1=4$ whose parameters are $[[24,17,5 ; 1]]_{5}$, $[[24,15,6 ; 1]]_{5},[[24,13,7 ; 1]]_{5},[[24,11,8 ; 1]]_{5}$.

\subsection{Length $n=q^{2}$}

Let $\mathcal{R S}(n-1, r)$ denote a RS code of length $n-1$ over $\mathbb{F}_{q^{2}}$ with parameters $[n-1, n-r, r]$. We extend $\mathcal{R S}(n-1, r)$ by adding an overall parity check, and denote the extended code by $\widehat{\mathcal{R S}}(n-1, r)$. Then $\widehat{\mathcal{R S}}(n-1, r)$ has parameters $[n, n-r, r+1]$. Let $\alpha$ be a primitive element of $\mathbb{F}_{q^{2}}$ and let $\left(\alpha_{1}, \alpha_{2}, \ldots, \alpha_{n}\right)=\left(0,1, \ldots, \alpha^{n-2}\right)$. Then the parity check matrix of $\widehat{\mathcal{R S}}(n-1, r)$ is given by

$$
H_{\widehat{\mathcal{R S}}(n-1, r)}=\left(\begin{array}{cccc}
1 & 1 & \cdots & 1 \\
\alpha_{1} & \alpha_{2} & \cdots & \alpha_{n} \\
\vdots & \vdots & \vdots & \vdots \\
\alpha_{1}^{r-1} & \alpha_{2}^{r-1} & \cdots & \alpha_{n}^{r-1}
\end{array}\right) .
$$

Lemma 7 If $q \leq r \leq 2 q-2$, then the rank of $H_{\widehat{\mathcal{R S}}(n-1, r)} H_{\widehat{\mathcal{R S}}(n-1, r)}^{\dagger}$ is equal to 1 .

Proof. It is easy to find that $1 \leq r \leq q-1 \Leftrightarrow \widehat{\mathcal{R S}}(n-1, r)^{\perp_{n}} \subseteq \widehat{\mathcal{R S}}(n-1, r)$ by [19, Lemma 8]. If $q \leq r \leq 2 q-2$, then we have

$$
\begin{aligned}
& H_{\widehat{\mathcal{R S}}(n-1, r)} H_{\widehat{\mathcal{R S}}(n-1, r)}^{\dagger} \\
= & \left(\begin{array}{cccc}
1 & 1 & \cdots & 1 \\
\alpha_{1} & \alpha_{2} & \cdots & \alpha_{n} \\
\vdots & \vdots & \vdots & \vdots \\
\alpha_{1}^{r-1} & \alpha_{2}^{r-1} & \cdots & \alpha_{n}^{r-1}
\end{array}\right) \cdot\left(\begin{array}{cccc}
1 & 1 & \cdots & 1 \\
\alpha_{1}^{q} & \alpha_{2}^{q} & \cdots & \alpha_{n}^{q} \\
\vdots & \vdots & \vdots & \vdots \\
\alpha_{1}^{q(r-1)} & \alpha_{2}^{q(r-1)} & \cdots & \alpha_{n}^{q(r-1)}
\end{array}\right)^{T} \\
= & \left(\begin{array}{cccccc}
0 & 0 & \cdots & 0 & \cdots & 0 \\
\vdots & \vdots & \vdots & \vdots & \vdots & \vdots \\
0 & 0 & \cdots & -1 & \cdots & 0 \\
\vdots & \vdots & \vdots & \vdots & \vdots & \vdots \\
0 & 0 & \cdots & 0 & \cdots & 0
\end{array}\right),
\end{aligned}
$$

where the "-1" in the $q$ th row and $q$ th column of matrix (7) is given by

$$
\alpha_{1}^{q^{2}-1}+\alpha_{2}^{q^{2}-1}+\ldots+\alpha_{n}^{q^{2}-1}=0+1+\ldots+1=-1 .
$$

The zero elements of matrix (7) are given by

$$
\begin{aligned}
1+1+\ldots+1 & =0, \\
\alpha_{1}^{r_{1}}+\alpha_{2}^{r_{1}}+\ldots+\alpha_{n}^{r_{1}} & =0, \\
\alpha_{1}^{q r_{2}}+\alpha_{2}^{q r_{2}}+\ldots+\alpha_{n}^{q r_{2}} & =0, \\
\alpha_{1}^{r_{1}+q r_{2}}+\alpha_{2}^{r_{1}+q r_{2}}+\ldots+\alpha_{n}^{r_{1}+q r_{2}} & =0,
\end{aligned}
$$


where $1 \leq r_{1}, r_{2} \leq r-1$, and then $r_{1}$ and $r_{2}$ are not equal to $q-1$ simultaneously. Therefore, the rank of $H_{\widehat{\mathcal{R S}}(n-1, r)} H_{\widehat{\mathcal{R S}}(n-1, r)}^{\dagger}$ is equal to 1 .

Combining Corollary 1 and Lemma 7, we can obtain the following EAQMDS code with length $q^{2}$.

Theorem 4 There exists an EAQMDS code with parameters $\left[\left[q^{2}, q^{2}-2 d+3, d ; 1\right]\right]_{q}$, where $q$ is a prime power, $q+1 \leq d \leq 2 q-1$ is an integer.

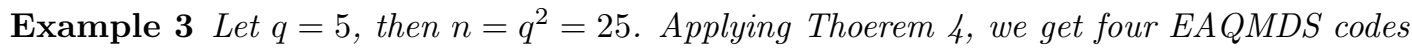
with minimum distance greater than $q=5$ whose parameters are $[[25,16,6 ; 1]]_{5},[[25,14,7 ; 1]]_{5}$, $[[25,12,8 ; 1]]_{5},[[25,10,9 ; 1]]_{5}$.

\subsection{EAQMDS codes that consume more than one maximally entangled states}

In 23, 24, 26, 27, many QMDS codes have been constructed based on negacyclic codes and constacyclic codes. If we introduce a certain amount of extra pre-shared entanglement in some special cases, we can get EAQMDS codes with larger minimum distance.

Let $q$ be an odd prime power and $n=\frac{q^{2}-1}{2}$. For $1 \leq j \leq n$, it is easy to see that the $q^{2}$-ary cyclotomic coset containing $2 j-1$ modulo $2 n$ has only one element $2 j-1$, i.e., $C_{2 j-1}=\{2 j-1\}$.

Lemma 8 Let $q$ be an odd prime power and $n=\frac{q^{2}-1}{2}$. Let $\mathcal{C}$ be a $q^{2}$-ary negacyclic code of length $n$ with defining set $Z=\cup_{j=-\delta_{1}}^{\delta_{2}} C_{2 j-1}$, where $1 \leq \delta_{1} \leq \frac{q-1}{2}-1$ and $\frac{q+1}{2} \leq \delta_{2} \leq q-1$, and let $H$ be the parity check matrix of $\mathcal{C}$ over $\mathbb{F}_{q^{2}}$, then $\operatorname{rank}\left(H H^{\dagger}\right)=2$.

Proof. We divide the defining set $Z$ of $\mathcal{C}$ into three mutually disjoint subsets, i.e., $Z=$ $Z_{1} \cup C_{-1} \cup Z_{2}$, where $Z_{1}=\cup_{j=1}^{\delta_{1}} C_{-2 j-1}$ and $Z_{2}=\cup_{j=1}^{\delta_{2}} C_{2 j-1}$. Let $\mathcal{C}_{1}$ and $\mathcal{C}_{2}$ be two $q^{2}$-ary negacyclic codes of length $n$ with defining sets $Z_{1}$ and $Z_{2}$, respectively. We know that $\mathcal{C}_{1}^{\perp_{h}} \subseteq \mathcal{C}_{1}$ and $\mathcal{C}_{2}^{\perp_{h}} \subseteq \mathcal{C}_{2}$ by [24, Lemma 3.1]. We show that $\mathcal{C}_{1}^{\perp_{h}} \subseteq \mathcal{C}_{2}$. Seeking a contradiction, we assume that $Z_{1} \cap Z_{2}^{-q} \neq \emptyset$ by Lemma 3. Then there exist $k$ and $l$, where $1 \leq k \leq \frac{q-1}{2}-1$ and $1 \leq l \leq q-1$, such that $-2 k-1=-q(2 l-1)(\bmod 2 n)$, which means that $q(2 l-1)-(2 k+1)=0$ $(\bmod 2 n)$. It follows that $q(2 l-1)-(2 k+1)=q^{2}-1$ since $2 \leq q(2 l-1)-(2 k+1) \leq 2 q^{2}-3 q-3$. However, there is $0 \leq 2 k=q(2 l-q-1) \leq q^{2}-3 q$. Then we have $k=0$ or $2 k \geq 2 q$, which are both contradictions. Therefore, we have $\mathcal{C}_{1}^{\perp_{h}} \subseteq \mathcal{C}_{2}$. Let the parity check matrices of $\mathcal{C}_{1}$ and $\mathcal{C}_{2}$ over $\mathbb{F}_{q^{2}}$ be $H_{1}$ and $H_{2}$, respectively, then we have $H_{1} H_{1}^{\dagger}=0, H_{2} H_{2}^{\dagger}=0$ and $H_{1} H_{2}^{\dagger}=0$. It is easy to see that $C_{-1} \cap C_{-1}^{-q}=\emptyset, C_{-1} \cap Z_{1}^{-q}=Z_{1} \cap C_{-1}^{-q}=\emptyset, C_{-1} \cap Z_{2}^{-q}=\{-1\}$ and $Z_{2} \cap C_{-1}^{-q}=\{q\}$, hence, $h_{-1} h_{-1}^{\dagger}=0, h_{-1} H_{1}^{\dagger}=0, H_{1} h_{-1}^{\dagger}=0, h_{-1} H_{2}^{\dagger}$ is a nonzero row vector and $H_{2} h_{-1}^{\dagger}$ is a nonzero column vector. Then the parity check matrix of $\mathcal{C}$ over $\mathbb{F}_{q^{2}}$ is given by $H=\left(\begin{array}{c}H_{1} \\ h_{-1} \\ H_{2}\end{array}\right)$, where $h_{-1}=\left(1, \eta^{-1}, \ldots, \eta^{-(n-1)}\right)$. Then we have

$$
H H^{\dagger}=\left(\begin{array}{ccc}
H_{1} H_{1}^{\dagger} & H_{1} h_{-1}^{\dagger} & H_{1} H_{2}^{\dagger} \\
h_{-1} H_{1}^{\dagger} & h_{-1} h_{-1}^{\dagger} & h_{-1} H_{2}^{\dagger} \\
H_{2} H_{1}^{\dagger} & H_{2} h_{-1}^{\dagger} & H_{2} H_{2}^{\dagger}
\end{array}\right)=\left(\begin{array}{ccc}
0 & 0 & 0 \\
0 & 0 & h_{-1} H_{2}^{\dagger} \\
0 & H_{2} h_{-1}^{\dagger} & 0
\end{array}\right) .
$$

It follows that the rank of $H H^{\dagger}$ is equal to 2.

Theorem 5 There exists an EAQMDS code with parameters $\left[\left[\frac{q^{2}-1}{2}, \frac{q^{2}-1}{2}-2 d+4, d ; 2\right]\right]_{q}$, where $q$ is an odd prime power, $\frac{q+1}{2}+2 \leq d \leq \frac{3}{2} q-\frac{1}{2}$. 
Proof. Consider the negacyclic code $\mathcal{C}$ over $\mathbb{F}_{q^{2}}$ of length $\frac{q^{2}-1}{2}$ with defining set $Z=$ $\cup_{j=-\delta_{1}}^{\delta_{2}} C_{2 j-1}$, where $0 \leq \delta_{1} \leq \frac{q-1}{2}-1$ and $\frac{q+1}{2} \leq \delta_{2} \leq q-1$. Then the defining set $Z$ which consists of $\delta_{1}+\delta_{2}+1$ consecutive odd integers is given by $\left\{-2 \delta_{1}-1,-2 \delta_{1}+\right.$ $\left.1, \ldots,-1,1, \ldots, 2 \delta_{2}-3,2 \delta_{2}-1\right\}$. Therefore, we have $\operatorname{dim} \mathcal{C}=\frac{q^{2}-1}{2}-\delta_{1}-\delta_{2}-1$. From the $\mathrm{BCH}$ bound for negacyclic codes in Lemma 2, the minimum distance of $\mathcal{C}$ is at least $\delta_{1}+\delta_{2}+2$. Then $\mathcal{C}$ has parameters $\left[\frac{q^{2}-1}{2}, \frac{q^{2}-1}{2}-\delta_{1}-\delta_{2}-1, \geq \delta_{1}+\delta_{2}+2\right]_{q^{2}}$. Combining Corollary 1. Lemma 8 and the EA-Singleton bound, we can obtain an EAQMDS code with parameters $\left[\left[\frac{q^{2}-1}{2}, \frac{q^{2}-1}{2}-2 \delta_{1}-2 \delta_{2}-2, \delta_{1}+\delta_{2}+2 ; 2\right]\right]_{q}$. Let $d=\delta_{1}+\delta_{2}+2$, we have $\frac{q+1}{2}+2 \leq d \leq \frac{3}{2} q-\frac{1}{2}$.

Example 4 Let $q=5$, then $n=\frac{q^{2}-1}{2}=12$. Applying Theorem 5 , we get three EAQMDS codes with parameters $[[12,6,5 ; 2]]_{5},[[12,4,6 ; 2]]_{5},[[12,2,7 ; 2]]_{5}$.

Let $t \geq 3$ be an odd integer and let $q$ be an odd prime power with $t \mid(q+1)$. Denote $n=\frac{q^{2}-1}{t}$. Let $\lambda \in \mathbb{F}_{q^{2}}$ be a primitive $t$-th root of unity. It is easy to see that every $q^{2}$ cyclotomic coset modulo tn contains only one element. In [26, 27], $q$-ary QMDS codes of length $n=\frac{q^{2}-1}{t}$ have been constructed from Hermitian dual-containing $\lambda$-constacyclic MDS codes. Based on the $\lambda$-constacyclic MDS codes, and if we introduce a certain amount of extra pre-shared entanglement, we can get EAQMDS codes with larger minimum distance compared with QMDS codes in 26, 27] of length $n=\frac{q^{2}-1}{t}$. Let $\mathcal{C}$ be a $\lambda$-constacyclic code of length $n$ over $\mathbb{F}_{q^{2}}$ with defining set

$$
Z=\cup_{i=-\delta_{1}}^{\delta_{2}} C_{1+t\left(\frac{(t-1)(q-1)-2}{2 t}+i\right)},
$$

where $C_{1+t\left(\frac{(t-1)(q-1)-2}{2 t}+i\right)}=\left\{1+t\left(\frac{(t-1)(q-1)-2}{2 t}+i\right)\right\}$ for $-\delta_{1} \leq i \leq \delta_{2}, \frac{(t-1)(q+1)}{2 t} \leq \delta_{1} \leq$ $\frac{(t+1)(q+1)}{2 t}-2$ and $\frac{(t-1)(q+1)}{2 t} \leq \delta_{2} \leq \frac{(t+1)(q+1)}{2 t}-2$.

Lemma 9 Let $t \geq 3$ be an odd integer and let $q$ be an odd prime power with $t \mid(q+1)$. Denote $n=\frac{q^{2}-1}{t}$. Let $\mathcal{C}$ be a $q^{2}$-ary $\lambda$-constacyclic code of length $n$ with defining set $Z=$ $\cup_{i=-\delta_{1}}^{\delta_{2}} C_{1+t\left(\frac{(t-1)(q-1)-2}{2 t}+i\right)}$, where $\frac{(t-1)(q+1)}{2 t} \leq \delta_{1} \leq \frac{(t+1)(q+1)}{2 t}-2$ and $\frac{(t-1)(q+1)}{2 t} \leq \delta_{2} \leq$ $\frac{(t+1)(q+1)}{2 t}-2$, and let $H$ be the parity check matrix of $\mathcal{C}$ over $\mathbb{F}_{q^{2}}$, then rank $\left(H H^{\dagger}\right)=t$.

Proof. Denote $s=(t-1) / 2$. We can divide the defining set $Z$ of $\mathcal{C}$ into three mutually disjoint subsets, i.e., $Z=Z_{1} \cup C_{s(q-1)} \cup Z_{2}$, where $Z_{1}=\cup_{j=1}^{\delta_{1}} C_{1+t\left(\frac{(t-1)(q-1)-2}{2 t}-j\right)}$ and $Z_{2}=$ $\cup_{k=1}^{\delta_{2}} C_{1+t\left(\frac{(t-1)(q-1)-2}{2 t}+k\right)}$. Let $\mathcal{C}_{1}$ and $\mathcal{C}_{2}$ be two $q^{2}$-ary $\lambda$-constacyclic codes of length $n$ with defining sets $Z_{1}^{2 t}$ and $Z_{2}$, respectively. Let the parity check matrices of $\mathcal{C}_{1}$ and $\mathcal{C}_{2}$ over $\mathbb{F}_{q^{2}}$ be $H_{1}$ and $H_{2}$, respectively. Then the parity check matrix of $\mathcal{C}$ is given by $H=\left(\begin{array}{c}H_{1} \\ h_{q-1} \\ H_{2}\end{array}\right)$, where $h_{q-1}=\left(1, \eta^{q-1}, \ldots, \eta^{(n-1)(q-1)}\right)$. From [26, Lemma 3.6] and [27, Lemma 4.1], there are $\mathcal{C}_{1}^{\perp_{h}} \subseteq \mathcal{C}_{1}$ and $\mathcal{C}_{2}^{\perp_{h}} \subseteq \mathcal{C}_{2}$, hence $H_{1} H_{1}^{\dagger}=0$ and $H_{2} H_{2}^{\dagger}=0$. It is easy to see that $C_{s(q-1)} \cap C_{s(q-1)}^{-q}=\{s(q-1)\}, Z_{1} \cap C_{s(q-1)}^{-q}=C_{s(q-1)} \cap Z_{1}^{-q}=\emptyset$ and $Z_{2} \cap C_{s(q-1)}^{-q}=$ $C_{s(q-1)} \cap Z_{2}^{-q}=\emptyset$, then there are $h_{-1} h_{-1}^{\dagger}=1+1+\cdots+1 \neq 0, H_{1} h_{-1}^{\dagger}=0, h_{-1} H_{1}^{\dagger}=0$ and $h_{-1} H_{2}^{\dagger}=0, H_{2} h_{-1}^{\dagger}=0$. Then we have

$$
H H^{\dagger}=\left(\begin{array}{ccc}
H_{1} H_{1}^{\dagger} & H_{1} h_{-1}^{\dagger} & H_{1} H_{2}^{\dagger} \\
h_{-1} H_{1}^{\dagger} & h_{-1} h_{-1}^{\dagger} & h_{-1} H_{2}^{\dagger} \\
H_{2} H_{1}^{\dagger} & H_{2} h_{-1}^{\dagger} & H_{2} H_{2}^{\dagger}
\end{array}\right)=\left(\begin{array}{ccc}
0 & 0 & H_{1} H_{2}^{\dagger} \\
0 & h_{-1} h_{-1}^{\dagger} & 0 \\
H_{2} H_{1}^{\dagger} & 0 & 0
\end{array}\right) .
$$


It follows that $\operatorname{rank}\left(H H^{\dagger}\right)=2 \operatorname{rank}\left(H_{1} H_{2}^{\dagger}\right)+1$. Next, we have to compute the rank of $H_{1} H_{2}^{\dagger}$. We determine the intersection of $Z_{1}$ and $Z_{2}^{-q}$. We assume that there exist $j$ and $k$, where $1 \leq j \leq \frac{(t+1)(q+1)}{2 t}-2$ and $1 \leq k \leq \frac{(t+1)(q+1)}{2 t}-2$, such that $1+t\left(\frac{(t-1)(q-1)-2}{2 t}-j\right)=$ $-q\left(1+t\left(\frac{(t-1)(q-1)-2}{2 t}+k\right)\right)\left(\bmod q^{2}-1\right)$, which means that $t q k-t j=0\left(\bmod q^{2}-1\right)$. Since $\frac{t-1}{2} q+\frac{3 t-1}{2} \leq t q k-t j \leq \frac{t+1}{2} q^{2}-\frac{3 t-1}{2} q-t$, it follows that $t q k-t j \in\left\{q^{2}-1, \ldots, \frac{t-1}{2}\left(q^{2}-\right.\right.$ $1)\}$. Denote $t q k-t j=x_{t}\left(q^{2}-1\right)$, where $x_{t} \in\{1, \ldots, s\}$, then we have $\frac{x_{t}\left(q^{2}-1\right)+t}{t q} \leq k \leq$ $\frac{2 x_{t}\left(q^{2}-1\right)+(t+1) q-3 t+1}{2 t q}$. Note that $\frac{x_{t}(q+1)}{t}-1<\frac{x_{t}\left(q^{2}-1\right)+t}{t q} \leq k \leq \frac{2 x_{t}\left(q^{2}-1\right)+(t+1) q-3 t+1}{2 t q}<$ $\frac{x_{t}(q+1)}{t}+1$. It follows that $k=\frac{x_{t}(q+1)}{t}$ and $j=\frac{x_{t}(q+1)}{t}$ for $x_{t} \in\{1, \ldots, s\}$. Therefore, we have $Z_{1} \cap Z_{2}^{-q}=\left\{\frac{\left(t-2 x_{t}-1\right) q-2 x_{t}-t-1}{2 t} \mid x_{t}=1, \ldots, s\right\}$ and $\left|Z_{1} \cap Z_{2}^{-q}\right|=s$. We can redivide $Z_{1}$ and $Z_{2}$ into mutually disjoint subsets, respectively, then the rank of $H_{1} H_{2}^{\dagger}$ is equal to $s$. Therefore, $\operatorname{rank}\left(H H^{\dagger}\right)=2 \cdot s+1=t$.

Theorem 6 Let $t \geq 3$ be an odd integer and let $q$ be an odd prime power with $t \mid(q+1)$. Then, there exists an EAQMDS code with parameters $\left[\left[\frac{q^{2}-1}{t}, \frac{q^{2}-1}{t}-2 d+t+2, d ; t\right]\right]_{q}$, where $\frac{(t-1)(q+1)}{t}+2 \leq d \leq \frac{(t+1)(q+1)}{t}-2$.

Proof. Let $\mathcal{C}$ be a $\lambda$-constacyclic code over $\mathbb{F}_{q^{2}}$ of length $\frac{q^{2}-1}{t}$ with defining set $Z=$ $\cup_{i=-\delta_{1}}^{\delta_{2}} C_{1+t\left(\frac{(t-1)(q-1)-2}{2 t}+i\right)}$, where $\frac{(t-1)(q+1)}{2 t} \leq \delta_{1} \leq \frac{(t+1)(q+1)}{2 t}-2$ and $\frac{(t-1)(q+1)}{2 t} \leq \delta_{2} \leq$ $\frac{(t+1)(q+1)}{2 t}-2$. Note that $\operatorname{dim} \mathcal{C}=\frac{q^{2}-1}{t}-\delta_{1}-\delta_{2}-1$, and the minimum distance of $\mathcal{C}$ is at least $\delta_{1}+\delta_{2}+2$ by the $\mathrm{BCH}$ bound for constacyclic codes in Lemma 2. Then $\mathcal{C}$ has parameters $\left[\frac{q^{2}-1}{t}, \frac{q^{2}-1}{t}-\delta_{1}-\delta_{2}-1, \geq d\right]_{q^{2}}$, where $d=\delta_{1}+\delta_{2}+2$. Combining Corollary 1 , Lemma 9 and the EA-Singleton bound, we can obtain an EAQMDS code with parameters $\left[\left[\frac{q^{2}-1}{t}, \frac{q^{2}-1}{t}-2 \delta_{1}-2 \delta_{2}+1, d ; t\right]\right]_{q}$, where $\frac{(t-1)(q+1)}{t}+2 \leq d \leq \frac{(t+1)(q+1)}{t}-2$.

Example 5 Let $t=3$ and $q=11$, then $n=\frac{q^{2}-1}{3}=40$. We get five EAQMDS codes with $p a$ rameters $[[40,25,10 ; 3]]_{11},[[40,23,11 ; 3]]_{11},[[40,21,12 ; 3]]_{11},[[40,19,13 ; 3]]_{11},[[40,17,14 ; 3]]_{11}$.

Example 6 Let $t=5$ and $q=19$, then $n=\frac{q^{2}-1}{5}=72$. We get five EAQMDS codes with parameters $[[72,43,18 ; 5]]_{19}$, $[[72,41,19 ; 5]]_{19}$, $[[72,39,20 ; 5]]_{19},[[72,37,21 ; 5]]_{19},[[72,35,22 ; 5]]_{19}$. Example 7 Let $t=7, q=27$, then $n=\frac{q^{2}-1}{7}=104$. We get five EAQMDS codes with parameters $[[104,61,26 ; 7]]_{27},[[104,59,27 ; 7]]_{27},[[104,57,28 ; 7]]_{27},[[104,55,29 ; 7]]_{27},[[104,53,30$; $7]]_{27}$.

\section{Conclusion}

We have constructed several classes of entanglement-assisted quantum MDS (EAQMDS) codes based on classical MDS codes for some certain code lengths. We list a comparison in Table 1 between EAQMDS codes constructed in this paper and the standard QMDS codes. Compared with the known QMDS codes of the same length, these EAQMDS codes have much larger minimum distance upper limit by exploiting one or more pre-shared maximally entangled states. In the future work, we look forward to getting more $q$-ary EAQMDS codes with minimum distance greater than $q+1$.

\section{Acknowledgements}

The authors are grateful to the Editor and the anonymous referee for their constructive comments and valuable suggestions. The first author J. Fan thanks the financial support 
Table 1. Comparison between EAQMDS codes and standard QMDS codes

\begin{tabular}{|c|c|c|c|}
\hline Length & $q$-ary EAQMDS codes & $q$-ary QMDS codes & Reference \\
\hline$q^{2}+1$ & $\begin{array}{c}{\left[\left[q^{2}+1, q^{2}-2 d+4, d ; 1\right]\right],} \\
2 \leq d \leq 2 q, d \text { even }\end{array}$ & $\begin{array}{c}{\left[\left[q^{2}+1, q^{2}-2 d+3, d\right]\right]} \\
2 \leq d \leq q+1\end{array}$ & $\begin{array}{l}17,21] \\
22,23\end{array}$ \\
\hline$q^{2}$ & $\begin{array}{c}{\left[\left[q^{2}, q^{2}-2 d+3, d ; 1\right]\right]} \\
q+1 \leq d \leq 2 q-1\end{array}$ & $\begin{array}{c}{\left[\left[q^{2}, q^{2}-2 d+2, d\right]\right]} \\
2 \leq d \leq q\end{array}$ & 15, 19 \\
\hline$q^{2}-1$ & $\begin{array}{c}{\left[\left[q^{2}-1, q^{2}-2 d+2, d ; 1\right]\right]} \\
2 \leq d \leq 2 q-2\end{array}$ & $\begin{array}{c}{\left[\left[q^{2}-1, q^{2}-2 d+1, d\right]\right]} \\
2 \leq d \leq q-1\end{array}$ & 15], 19] \\
\hline$\frac{q^{2}-1}{2}, q$ odd & $\begin{array}{l}{\left[\left[\frac{q^{2}-1}{2}, \frac{q^{2}-1}{2}-2 d+4, d ; 2\right]\right]} \\
(q+1) / 2+2 \leq d \leq \frac{3}{2} q-\frac{1}{2}\end{array}$ & $\begin{array}{c}{\left[\left[\frac{q^{2}-1}{2}, \frac{q^{2}-1}{2}-2 d+2, d\right]\right],} \\
2 \leq d \leq q\end{array}$ & 24,26 \\
\hline $\begin{array}{c}\frac{q^{2}-1}{t}, q \text { odd } \\
t \mid(q+1) \\
t \geq 3 \text { odd }\end{array}$ & $\begin{array}{c}{\left[\left[\frac{q^{2}-1}{t}, \frac{q^{2}-1}{t}-2 d+t+2, d ; t\right]\right],} \\
\frac{(t-1)(q+1)}{t}+2 \leq d \leq \frac{(t+1)(q+1)}{t}-2\end{array}$ & $\begin{array}{c}{\left[\left[\frac{q^{2}-1}{t}, \frac{q^{2}-1}{t}-2 d+2, d\right]\right]} \\
2 \leq d \leq \frac{(t+1)(q+1)}{2 t}-1\end{array}$ & 26, 27. \\
\hline
\end{tabular}

from China Scholarship Council (CSC, No. 201406090079). J. Fan thanks Dr. Bocong Chen for the helpful communication. This work was supported by the National Natural Science Foundation of China (Grant No. 61170321), the Specialized Research Fund for the Doctoral Program of Higher Education (Grant No. 20110092110024), the Natural Science Foundation of Jiangsu Province (Grant No. BK20140823), China Postdoctoral Science Foundation (Grant No. 2013M531353) and the Scientific Research Innovation Plan for College Graduates of Jiangsu Province (Grant No. CXZZ13_0105). This work was partially carried out when the first author was visiting the School of Electrical and Information Engineering, University of Sydney. He thanks the school for its hospitality.

\section{References}

1. A. R. Calderbank, E. M. Rains, P. Shor, and N. J. Sloane, "Quantum error correction via codes over GF(4)," IEEE Trans. Inform. Theory, vol. 44, no. 4, pp. 1369-1387, 1998.

2. D. Gottesman, "Stabilizer codes and quantum error correction," Ph.D. dissertation, California Institute of Technology, 1997.

3. A. Ketkar, A. Klappenecker, S. Kumar, and P. K. Sarvepalli, "Nonbinary stabilizer codes over finite fields," IEEE Trans. Inform. Theory, vol. 52, no. 11, pp. 4892-4914, 2006.

4. T. Brun, I. Devetak, and M.-H. Hsieh, "Correcting quantum errors with entanglement," Science, vol. 314, no. 5798, pp. 436-439, 2006.

5. M.-H. Hsieh, T. A. Brun, and I. Devetak, "Entanglement-assisted quantum quasicyclic low-density parity-check codes," Phys. Rev. A, vol. 79, no. 3, p. 032340, 2009.

6. M.-H. Hsieh, W.-T. Yen, and L.-Y. Hsu, "High performance entanglement-assisted quantum LDPC codes need little entanglement," IEEE Trans. Inform. Theory, vol. 57, no. 3, pp. 1761-1769, 2011.

7. Y. Fujiwara, D. Clark, P. Vandendriessche, M. De Boeck, and V. D. Tonchev, "Entanglementassisted quantum low-density parity-check codes," Phys. Rev. A, vol. 82, no. 4, p. 042338, 2010.

8. Y. Fujiwara and V. D. Tonchev, "A characterization of entanglement-assisted quantum low-density parity-check codes," IEEE Trans. Inform. Theory, vol. 59, no. 6, pp. 3347-353, 2013.

9. M. M. Wilde, M.-H. Hsieh, and Z. Babar, "Entanglement-assisted quantum turbo codes," IEEE Trans. Inform. Theory, vol. 60, no. 2, pp. 1203-1222, 2014.

10. L.-D. Lü and R. Li, "Entanglement-assisted quantum codes constructed from primitive quaternary BCH codes," Int. J. Quantum Inf., vol. 12, no. 03, p. 1450015, 2014.

11. D. A. Lidar and T. A. Brun, Quantum error correction. Cambridge: Cambridge University Press, 
2013.

12. M. M. Wilde, Quantum Information Theory. Cambridge: Cambridge University Press, 2013.

13. F. J. MacWilliams and N. J. A. Sloane, The Theory of Error-Correcting Codes. Amsterdam: The Netherlands: North-Holland, 1981.

14. M. Grassl, W. Geiselmann, and T. Beth, "Quantum Reed-Solomon codes," in Applied Algebra, Algebraic Algorithms and Error-correcting Codes. Springer, 1999, pp. 231-244.

15. Z. Li, L.-J. Xing, and X.-M. Wang, "Quantum generalized Reed-Solomon codes: Unified framework for quantum maximum-distance-separable codes," Phys. Rev. A, vol. 77, no. 1, p. 012308, 2008.

16. M. Grassl and M. Roetteler, "Quantum MDS codes over small fields," in Proc. IEEE Int. Symp. Inf. Theory, Hong Kong, June 2015, pp. 1104-1108.

17. L. Jin and C. Xing, "A construction of new quantum MDS codes," IEEE Trans. Inform. Theory, vol. 60, no. 5, pp. 2921-2925, 2014.

18. M. Rötteler, M. Grassl, and T. Beth, "On quantum MDS codes," in Proc. IEEE Int. Symp. Inf. Theory, Chicago, IL, USA, June 2004, pp. 356-356.

19. M. Grassl, T. Beth, and M. Roetteler, "On optimal quantum codes," Int. J. Quantum Inf., vol. 2, no. 01, pp. 55-64, 2004.

20. R. Li and Z. Xu, "Construction of $[[n, n-4,3]]_{q}$ quantum codes for odd prime power $q$," Phys. Rev. A, vol. 82, no. 5, p. 052316, 2010.

21. L. Jin, S. Ling, J. Luo, and C. Xing, "Application of classical hermitian self-orthogonal MDS codes to quantum MDS codes," IEEE Trans. Inform. Theory, vol. 56, no. 9, pp. 4735-4740, 2010.

22. G. G. La Guardia, "New quantum MDS codes," IEEE Trans. Inform. Theory, vol. 57, no. 8, pp. 5551-5554, 2011.

23. X. Kai and S. Zhu, "New quantum MDS codes from negacyclic codes," IEEE Trans. Inform. Theory, vol. 59, no. 2, pp. 1193-1197, 2013.

24. X. Kai, S. Zhu, and P. Li, "Constacyclic codes and some new quantum MDS codes," IEEE Trans. Inform. Theory, vol. 60, no. 4, pp. 2080-2086, 2014.

25. G. Zhang and B. Chen, "New quantum MDS codes," Int. J. Quantum Inf., vol. 12, no. 04, 2014.

26. L. Wang and S. Zhu, "New quantum MDS codes derived from constacyclic codes," Quantum Inf. Process., vol. 14, no. 3, pp. 881-889, 2015.

27. B. Chen, S. Ling, and G. Zhang, "Application of constacyclic codes to quantum MDS codes," IEEE Trans. Inform. Theory, vol. 61, no. 3, pp. 1474-1484, 2015.

28. E. Berlekamp, Algebraic Coding Theory. New York, McGraw-Hill, 1968.

29. M. M. Wilde and T. A. Brun, "Optimal entanglement formulas for entanglement-assisted quantum coding," Phys. Rev. A, vol. 77, no. 6, p. 064302, 2008.

30. A. Krishna and D. V. Sarwate, "Pseudocyclic maximum-distance-separable codes," IEEE Trans. Inform. Theory, vol. 36, no. 4, pp. 880-884, 1990.

31. M. A. Nielsen and I. L. Chuang, Quantum Computation and Quantum Information. Cambridge: Cambridge University Press, 2000. 